\title{
Childhood B Lymphoblastic Lymphoma
}

National Cancer Institute

\section{Source}

National Cancer Institute. Childhood B Lymphoblastic Lymphoma. NCI Thesaurus. Code C7209.

A B lymphoblastic lymphoma that occurs during childhood. 